\title{
StepByStep: Design of an Interactive Pictorial Activity Game for Teaching Generalization Skills to Children with Autism
}

\author{
Alberto Gruarin $^{1}$, Michel A. Westenberg ${ }^{2}$, and Emilia I. Barakova ${ }^{1}$ \\ 1 Industrial Design Department, Eindhoven University of Technology \\ 2 Department of Mathematics and Computer Science, Eindhoven University of Technology \\ a.gruarin@student.tue.nl, \{m.a.westenberg,e.i.barakova\}atue.nl
}

\begin{abstract}
Translating acquired behavioral skills from training environments to daily-life situations is difficult for children with autism. This study introduces the StepByStep platform, in which pictorial activity schedules are implemented. Design decisions that increase the sameness in the environment and the comfort of the children were implemented to isolate the learning task and promote the training of generalization skills. Differently from existing visual scheduling systems, we use photographs of the child that is trained instead of general activity pictures. The design promotes the features of easy individualization of the training and of playfulness while learning. StepByStep was used by one participant in a pilot study, who showed behavior acquisition and translation to real life situations.
\end{abstract}

Keywords: Interactive game design, training generalization skills, assistive technology, pictorial activity, personalized training for children with ASD, visual scheduling.

\section{Introduction}

Generalization is the ability to transfer a learned behavior to another similar or related activity or situation, and is considered to be a difficult task for children with autism spectrum disorders (ASD). It is a complex task that can be divided in subtasks which include learning the right sequence of actions, understanding self-referential (emotionally non-neutral) concepts, and possibly imitation as a social learning mechanism.

The existing procedures for behavioral training of individuals with autism have been successful in structured and controlled environments [4]5]. In these situations, both the behavior and the stimulus that sets the occasion for learning are clearly presented to the child by an instructor. Moreover, the structured environment does not present other possible cues which can distract the child. In daily-life contexts, however, a child is usually exposed to various stimuli which can all set an occasion for social learning and generalization. Due to the difficulties these children have in dealing with multiple cues, isolating the stimulus that needs to be learned may help translating the learned behavior from training environments into real life. This design study implements a specific context for the behavior which should be learned by the child; the training is meant to present and teach a skill within the environment familiar to the child. In addition, we 
attempt to utilize the benefits of playfulness during training. The design is meant to give the child the impression that he/she is playing rather than learning, and it aims to decrease the perception of a starting and an ending time, usually associated with a therapy schedule. In particular, we propose an interactive game with physical objects which can be more engaging for the child. Behavioral training through interactive games has been used in the past years to reduce the stress of learning different skills [24].

Children with ASD generally tend to stick with sameness [8], which usually results in problematic behavioral responses to changes in daily routines. In this context, the literature shows that children with autism may benefit from visual support, especially when comprehending changes throughout the day [9]. Cihak [6] showed that both video modeling [4|11] and pictorial activity schedules provide benefits for autonomy and independence during the transition, reducing at the same time negative behavioral responses due to anxiety or aggressiveness. Activity schedules are visual support systems that combine photographs, images, or drawings in a sequential format to represent targeted sequences. Although they are an established and often used support for young people with autism, they mostly make use of paper sequences [12]. Creation and use of visual sequences that show the child's schedule can demand much time and it can be difficult to record data to track progress [10]. The design of vSked, a computerized visual scheduling system for interaction and collaboration, addresses these problems [10].

In the present study, we build on the concept of computerized activity schedules. However, we pursue different learning goals: teaching generalization skills to children with autism. We use the familiar home environment of the child, his/her mother as a trainer and an element that to our knowledge has not been used in other designs, namely pictures of the child himself/herself performing the actions of the behavioral sequence to be learned. These design decisions help to increase the familiarity of the child with the context and the event expressed in the pictures, and also to make the interpretation of the presented event unique. Since pictorial sequences are static, the child's attention can move from one image to another without losing any information at any time. This characteristic makes pictorial sequences also more effective for children who are easily distracted [1].

Regarding the content of the pictorial activity schedule, the child's personal representation in the images help him/her to interpret and acquire the meaning of the pictures. In general, autistic children show a lack of "theory of mind", i.e. they have difficulties in understanding others' mental states, beliefs and desires [3]. Corcoran \& Frith [7] suggested that understanding others' thoughts relies partially on the ability to recall autobiographical memories. These studies inspired the decision to implement pictures of the children themselves and of their own activities into designed game sequences, avoiding translation of others' actions to an own schedule. In addition, the children are assisted by recognizing themselves in the sequences. Recalling personal memories would, then, help them to perform the acquired behavior in real life.

To summarize, the designed game StepByStep implements activity schedules into behavioral training, presenting behavior in known context and environment and through picture sequences of the particular child performing these actions. The expectation is that these recognizable features will help the child to more easily learn and generalize this behavior to a real-life situation. 


\section{Design and Proof-of-Concept Validation}

Game Concept. StepByStep consists of two parts: a tangible carpet used as a controller, which interacts with the software game installed on a computer. The tangible carpet consists of 6 colorful pads with embedded buttons, on which the child can press or step (see Fig. 1(a)). The carpet is intentionally designed large sized to promote more physical activity. The game setting is as follows: pictorial activity sequences have to be reordered by the child. Every time a level starts, the sequence images are presented in a random order and assigned to a colored box at the top of the screen. By pressing the corresponding colored carpet button, the child is asked to construct the correct sequence order. If the answer is correct, an audio feedback will play a predefined tone once, and the software will move the image to the correct sequence position at the bottom of the screen (see Fig. 1(b)). In this way, visual feedback is provided to attract the child's attention both on the remaining images and on the already correct ones. If the answer is wrong, a different tone is continuously played until the child presses a button. The answer feedback is intentionally designed to avoid negative responses by the software.

The pictorial activity sequences are uploaded on the computer by the parents, perhaps together with a therapist, in order to direct the behavioral training to specific learning goals. In this way, the parents are able to control the amount of information provided to children with new sequences, balancing them with their child's abilities in integration and acquisition of new behaviors.

StepByStep Technical Specifications. StepByStep includes both hardware and software sections, which are designed on the Arduino microcontroller platform and Processing software, respectively.

The carpet is built with white eco-friendly leather onto which six eco-friendly leather colored sheets are sewn; inside this white folder, six pressure mats are positioned below the six colored sheets. These sensors, once pressed by the child, act as a switch for Arduino, which consequently is able to detect exactly which sensor is pressed. A threshold is set by Arduino in order to determine the minimum amount of pressure the child should apply to the sensor, to avoid that the carpet responds to small forces. When Arduino detects the signal from the pressure sensor, it sends this information to the software game controller for further handling.

The game controller is implemented in Processing to allow maximum platform independence and flexibility. The game resides on the computer, and the carpet merely acts as a game interface. This setup allows easy extensions to other input devices, such as keyboard or mouse, but also to touch interfaces as provided by tablet computers or smart phones. New image sequences can be added by the parents, simply by putting the corresponding pictures (minimally three and maximally six) in a pre-specified place and by providing a single text file that contains the filenames of the pictures in correct order. At the beginning of each level, the game presents a dialog box from which a desired training sequence can be chosen. The number of pictures in the sequence is communicated to Arduino, which initializes the sensors of the carpet, and disables the ones that are not involved in the sequence to avoid that the child receives feedback that is not meaningful. After loading the sequence, the images are permuted using a variant of the Fisher-Yates shuffling algorithm and linked to the carpet sensors. 


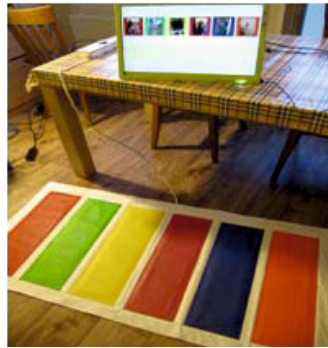

(a)

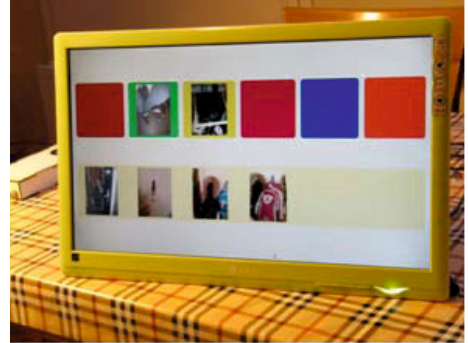

(b)

Fig. 1. (a) StepByStep design. (b) Screenshot during game play: [top] random image sequence and correspondence to the colored buttons; [bottom] partially ordered image sequence.

During game play, the child presses the sensors on the carpet. Arduino communicates the received input from this sensor to the game software, which checks if the child's answer is correct or wrong. This answer is sent back to Arduino which will play a sound depending on the outcome as described previously.

Experimental Procedure. The design and the proof-of-concept user test were performed with a Dutch female child aged 8. She was diagnosed to belong to the Pervasive Developmental Disorder Not Otherwise Specified (PDD-NOS) group, specifically to the high-functioning subgroup (whose symptoms largely overlap that of Asperger syndrome), but presenting language delays and mild cognitive impairments. The study took place at the child's home throughout the entire experimental period. Informed consent to take part to the test was obtained from the parents.

Two pictorial activity sequences were tested by the participant. Both sequences represented behaviors that the participant did not perform alone. Sequence 1 refers to coming back home from school, where she was required to open the front door, hang up the bag and then the jacket, take off the shoes and store them in a shoe rack. Sequence 2 represents what she should do if a nightmare occurs: turn on the room light, go up the stairs, and wake up her mother asking for help. The pictures for the two sequences were taken by the participant's mother, who involved the child and explained her the meaning and context of the pictures. The photographed environment was the participant's house, and the child and her personal objects appear in the image sequences.

During a three weeks span on regular intervals, the participant played StepByStep 7 times for 15 minutes. In each session, both sequences were presented to the child. The experimenter was present only in the first training together with the participant's mother, who was instructed how to observe the child's emotional state and behavior during the next trainings. An indirect observation method was chosen for this study; the participant's mother took on the observer role in the research due to her confidence with autistic children and her experience on understanding small emotional and behavioral changes of her child. Additionally, children with autism are largely affected by the presence of new individuals, which can lead to stress and anxiety and will affect their responses to the game. After all sessions, an interview with the participant's mother was held to collect data. 


\section{Results}

The participant showed enjoyment and willingness to play with StepByStep during all sessions. Children with autism typically refuse activities which do not match their preferences, and they tend to abandon them after a certain time span. In this specific study, since repetitive use is crucial for acquiring behaviors and translating them into daily life, it would also be difficult to achieve any valuable results if the child did not like the game or felt forced to play with StepByStep. Furthermore, the participant showed enjoyment in experimenting with the carpet by finding new ways for moving on it, and she used the carpet as a means to express her emotional state (for example, she would step back from the carpet after a mistake and then slowly push the button corresponding to the new answer trial).

After the training period, the participant was able to wake up her mother for help when a nightmare occurred (Sequence 2). Hence, she showed the ability to translate the pictorial sequence learned during game play to a real-life situation. In addition, the mother reported a conversation between the child and her therapist, where the child told that, in total, three nightmares occurred during the training period and that, since she played with the game, she was aware of the correct behavior to show in that situation. She asked for help (i.e. performed the sequence of actions for real) only the first time when the nightmare occurred. The mother thinks that during the other two occurrences, being aware of the correct response to a nightmare was already enough to reduce fear and anxiety of the child without asking for her mother's help.

The child could not yet translate the behavior from Sequence 1 (coming back from school) to real life. Most of the training took place during Christmas holidays, however, which had very few opportunities for the child to perform the intended behavior.

\section{Discussion}

Pictorial activity sequences provided a useful educational support for teaching behavioral responses. The two features of the pictures specific of this study, namely photographs of the child and her personal environment, showed to enhance this teaching support. According to the participant, being an actor for recording the sequences was perceived as part of the game, hence a playful activity. Playfulness decreased the perception of being engaged in learning but did not decrease the effect of learning. The child managed to acquire a specific response within the performance context, and was able in one case to translate this knowledge in real life. The child herself being on the pictures prevented the participant from interpreting other individual's intentions through the pictorial sequences; adding a personal environment as a context for learning the desired behavior resulted in both behavior acquisition and recall from autobiographical memories when needed. If only the response itself would have been taught, the child would have had to spontaneously add the correct context during real life. This requires good context interpretation abilities, which are usually not mastered by children with ASD.

StepByStep was evaluated with one participant for a time period of three weeks. Involving more participants of various chronological age groups and different ASD conditions is planned to further validate the design. Further research should be done to define 
a balance between new information provided by new sequences and training periods according to the individual abilities of the child. The present study only touches upon the effects of playing with StepByStep for behavioral training in ordering sequences of daily events and generalization/translation of newly learned behavioral sequences to daily routine. Generalization over contexts by children with autism might be further explored, by evaluating if StepByStep can successfully train behavioral sequences that have been learned at school or other non-domestic environments.

Acknowledgments. The authors thank the family who participated passionately throughout the research and M. van Dijk, therapist at Expertise center Kentalis (Eindhoven, The Netherlands), for her support and cooperation in the design phase.

\section{References}

1. Alberto, P.A., Cihak, D.F., Gama, R.I.: Use of static picture prompts versus video modeling during simulation instruction. Research in Developmental Disabilities 26(4), 327-339 (2005)

2. Barakova, E., Gillessen, J., Feijs, L.: Social training of autistic children with interactive intelligent agents. Journal of Integrative Neuroscience 8(01), 23-34 (2009)

3. Baron-Cohen, S., Leslie, A.M., Frith, U.: Does the autistic child have a "theory of mind"? Cognition 21(1), 37-46 (1985)

4. Brok, J.C.J., Barakova, E.I.: Engaging autistic children in imitation and turn-taking games with multiagent system of interactive lighting blocks. In: Yang, H.S., Malaka, R., Hoshino, J., Han, J.H. (eds.) ICEC 2010. LNCS, vol. 6243, pp. 115-126. Springer, Heidelberg (2010)

5. Brown, A.K., Brown, J.L., Poulson, C.L.: Discriminating which fork to use: Teaching selective imitation to people with autism. Research in Autism Spectrum Disorders 2(2), 199-208 (2008)

6. Cihak, D.F.: Comparing pictorial and video modeling activity schedules during transitions for students with autism spectrum disorders. Research in Autism Spectrum Disorders 5(1), 433-441 (2011)

7. Corcoran, R., Frith, C.D.: Autobiographical memory and theory of mind: evidence of a relationship in schizophrenia. Psychological Medicine (33), 897-905 (2003)

8. Heflin, L.J., Alaimo, D.F.: Students With Autism Spectrum Disorders: Effective Instructional Practices. Prentice Hall (2006)

9. Heflin, L.J., Simpson, R.L.: Interventions for children and youth with autism prudent choices in a world of exaggerated claims and empty promises. Part I: Intervention and treatment option review. Focus on Autism and Other Developmental Disabilities 13(4), 194-211 (1998)

10. Hirano, S.H., Yeganyan, M.T., Marcu, G., Nguyen, D.H., Boyd, L.A., Hayes, G.R.: vSked: evaluation of a system to support classroom activities for children with autism. In: Proceedings of the SIGCHI Conference on Human Factors in Computing Systems, pp. 1633-1642. ACM (2010)

11. Qi, C.H., Lin, Y.L.: Quantitative analysis of the effects of video modeling on social and communication skills for children with autism spectrum disorders. Procedia - Social and Behavioral Sciences (46), 4518-4523 (2012)

12. Savner, J.L., Hyles, B.S.: Making visual supports work in the home and community: Strategies for individuals with autism and Asperger Syndrome. AAPC Publishing (2000) 